(6) OPEN ACCESS

\title{
The Diabetes Visual Function Supplement Study (DiVFuSS)
}

\author{
A Paul Chous, ${ }^{1}$ Stuart P Richer, ${ }^{2}$ Jeffry D Gerson, ${ }^{3}$ Renu A Kowluru ${ }^{4}$
}

'Private Practice, Tacoma, Washington, USA

${ }^{2}$ Captain James A Lovell Federal Heath Care Center, North Chicago, Illinois, USA

${ }^{3}$ Private Practice, Olathe, Kansas, USA

${ }^{4}$ Kresge Eye Institute, Wayne State University, Detroit, Michigan, USA

Correspondence to Dr A Paul Chous, FAAO 6720 Regents Blvd., Tacoma, WA 98466, USA; dr.chous@diabeticeyes.com

Received 18 December 2014 Revised 8 April 2015 Accepted 26 May 2015 Published Online First 18 June 2015
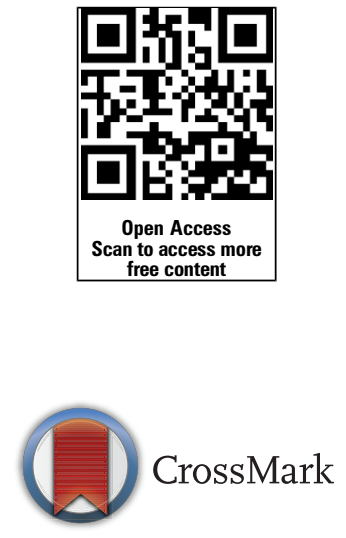

To cite: Chous AP, Richer SP, Gerson JD, et al. $\mathrm{Br} J$ Ophthalmol

2016;100:227-234.
ABSTRACT

Background Diabetes is known to affect visual function before onset of retinopathy (diabetic retinopathy (DR)). Protection of visual function may signal disruption of mechanisms underlying DR.

Methods This was a 6-month randomised, controlled clinical trial of patients with type 1 and type 2 diabetes with no retinopathy or mild to moderate non-proliferative retinopathy assigned to twice daily consumption of placebo or a novel, multi-component formula containing xanthophyll pigments, antioxidants and selected botanical extracts. Measurement of contrast sensitivity, macular pigment optical density, colour discrimination, 5-2 macular threshold perimetry, Diabetic Peripheral Neuropathy Symptoms, foveal and retinal nerve fibre layer thickness, glycohaemoglobin (HbA1c), serum lipids, 25-OH-vitamin D, tumour necrosis factor $\alpha$ (TNF-a) and high-sensitivity $C$ reactive protein (hsCRP) were taken at baseline and 6 months. Outcomes were assessed by differences between and within groups at baseline and at study conclusion using meand \pm SDs and $t$ tests $(p<0.05)$ for continuous variables.

Results There were no significant intergroup differences at baseline. At 6 months, subjects on active supplement compared with placebo had significantly better visual function on all measures ( $p$ values ranging from 0.008 to $<0.0001$ ), significant improvements in most serum lipids ( $p$ values ranging from 0.01 to 0.0004 ), hsCRP $(p=0.01)$ and diabetic peripheral neuropathy (Fisher's exact test, $p=0.0024$ ) No significant changes in retinal thickness, HbA1c, total cholesterol or TNF- $\alpha$ were found between the groups.

Conclusions This study provides strong evidence of clinically meaningful improvements in visual function, hsCRP and peripheral neuropathy in patients with diabetes, both with and without retinopathy, and without affecting glycaemic control.

Trial registration number www.ClinicalTrials.gov Identifier: NCT01646047

\section{INTRODUCTION}

Diabetic retinopathy (DR) remains a leading cause of visual impairment and blindness worldwide. Despite clinical trials showing that tighter control of blood glucose and blood pressure reduces the risk of microvascular diabetes complications, and despite tremendous advances in the clinical management of diabetic eye disease, rates of DR in the USA have increased by $89 \%$ over the last decade. ${ }^{1}$

Importantly, serious visual impairment associated with diabetes remains high. ${ }^{2}$ Recent estimates show nearly $5 \%$ of US adults with diabetes have sightthreatening DR (STR), with significantly higher rates among African, Latino and Native Americans. Although improving blood glucose control lowers the risk of DR and its progression, evidence shows that there is no level of average blood glucose (as reflected by glycosylated haemoglobin) that is totally protective against DR. The current clinical algorithm for delaying DR and preventing STR is earlier diagnosis of diabetes, tighter metabolic control, routine dilated retinal examinations and treatment (laser photocoagulation, intravitreal injections of anti-vascular endothelial growth factor (VEGF) agents and corticosteroids) if/when DR progresses to a level that threatens vision.

The Age-Related Eye Disease Study (AREDS) demonstrated that a nutritional supplement could positively influence progression of a visionthreatening eye disease, age-related macular degeneration. ${ }^{3}$ This begs the question as to whether nutritional supplements may benefit other eye diseases, including DR. Vitamins, minerals and other micronutrients have a variety of biological functions potentially beneficial in diabetes, serving as enzymatic cofactors mediating glucose homeostasis, as regulators of cell growth and differentiation, and as building blocks of antioxidant defence. Thus, there has been renewed interest in their potential for preventing or treating a host of diabetes complications. ${ }^{4}$

A number of investigators have shown that diabetes affects visual function prior to the development of DR detectable by ophthalmoscopy. This includes deficits in contrast, ${ }^{5}{ }^{6}$ visual field ${ }^{7} 8$ and colour vision sensitivity. ${ }^{9}{ }^{10}$ As such, amelioration of these visual function deficits may serve as an additional, useful biomarker for the onset and progression of retinopathy in patients with diabetes, yet no clinically evident DR, as well as those with established diabetes-induced retinal pathology. ${ }^{11-13}$

In Diabetes Visual Function Supplement Study (DiVFuSS), we prospectively examine visual function effects of a novel, multi-component nutritional supplement designed to disrupt established biological pathways in the genesis of DR (www. ClinicalTrials.gov Identifier: NCT01646047).

\section{SUBJECTS}

A total of $n=67$ adult subjects, 42 women $/ 25$ men, mean age 56.1 years $( \pm 13.2$ years) with either type 1 or type 2 diabetes, were recruited following informed consent under the Declarations of Helsinki. Sample size was based on previous published trials and differences in key visual function variables. Subjects were primarily pre-existing patients from a single optometric practice emphasising diabetes eye care, with $n=12$ referred from either a local retinal specialty or endocrinology practice. 
Table 1 Baseline characteristics of all subjects, and subjects randomised to active supplement or placebo

\begin{tabular}{|c|c|c|c|c|c|c|c|}
\hline & Age, years & DM duration, years & $\mathrm{HbA1c} \%$ & Gender & DM subtype & DR status & MVM use \\
\hline Total group $(n=67)$ & $56.1 \pm 13.2$ & $16 \pm 11.2$ & $7.2 \pm 1.1$ & $\begin{array}{l}42 \text { Female } \\
25 \text { Male }\end{array}$ & $\begin{array}{l}27 \mathrm{~T} 1 \mathrm{DM} \\
40 \mathrm{~T} 2 \mathrm{DM}\end{array}$ & $\begin{array}{l}\text { No } D R=37 \\
\text { Mild } D R=24 \\
\text { Mod DR=6 }\end{array}$ & $\begin{array}{l}23 \text { Yes } \\
44 \text { No }\end{array}$ \\
\hline DiVFuSS supplement $(n=39)$ & $53.5 \pm 14.6$ & $16 \pm 12.2$ & $7.1 \pm 1.0$ & $\begin{array}{l}26 \text { Female } \\
13 \text { Male }\end{array}$ & $\begin{array}{l}16 \text { T1DM } \\
23 \text { T2DM }\end{array}$ & $\begin{array}{l}\text { No } D R=24 \\
\text { Mild } D R=10 \\
\text { Mod } D R=5\end{array}$ & $\begin{array}{l}15 \text { Yes } \\
24 \text { No }\end{array}$ \\
\hline Placebo $(n=28)$ & $59.7 \pm 10.3$ & $16.1 \pm 9.9$ & $7.3 \pm 1.1$ & $\begin{array}{l}16 \text { Female } \\
12 \text { Male }\end{array}$ & $\begin{array}{l}11 \mathrm{~T} 1 \mathrm{DM} \\
17 \mathrm{~T} 2 \mathrm{DM}\end{array}$ & $\begin{array}{l}\text { No } D R=13 \\
\text { Mild } D R=14 \\
\text { Mod } D R=1\end{array}$ & $\begin{array}{l}8 \text { Yes } \\
20 \text { No }\end{array}$ \\
\hline
\end{tabular}

$p$ Values for all baseline characteristics were not statistically significantly different comparing supplemented vs placebo groups $(p<0.5)$.

DiVFuSS, Diabetes Visual Function Supplement Study; DM, diabetes mellitus; DR, diabetic retinopathy; MVM, multivitamin and mineral; T1DM, type 1 diabetes mellitus; T2DM, type 2

diabetes mellitus.

\section{Inclusion criteria}

Inclusion criteria include diabetes diagnosis $\geq 5$ years, best corrected visual acuity $\geq 20 / 30$ in each eye, with either no DR or mild to moderate non-proliferative DR (NPDR). Subjects with diabetic macular oedema (DMO) as determined by spectral domain optical coherence tomography (SDOCT) were eligible for participation, provided they did not meet diagnostic criteria for clinically significant DMO per Early Treatment Diabetic Retinopathy Study guidelines.

\section{Exclusion criteria}

Subjects with known sensitivity to any of the test ingredients, renal impairment, minors and non-English speakers/readers were excluded from participation. Of the 70 subjects initially enrolled, $n=67$ ( $n=134$ eyes) completed the trial (three subjects were lost to follow-up). Data pertaining to the three subjects who did not complete the study were excluded from both initial and final analyses. Twenty-seven $(n=27)$ patients with type 1 diabetes mellitus and $40(n=40)$ patients with type 2 diabetes mellitus were enrolled by the Primary Investigator from April 2012 to February 2014 and completed the trial; of these 67 subjects, $30(\mathrm{n}=30)$ had mild or moderate NPDR in at least one eye and $37(\mathrm{n}=37)$ had no DR in either eye at baseline. Subject characteristics are summarised in table 1 of Results Section.

\section{TEST FORMULA AND MASKING}

The DiVFuSS formula consists of vitamins C, D3 and E (d- $\alpha$ tocopherol), zinc oxide, eicosapentaenoic acid, docosahexaenoic acid, $\alpha$-lipoic acid (racemic mixture), coenzyme Q10, mixed tocotrienols/tocopherols, zeaxanthin, lutein, benfotiamine, $\mathrm{N}$-acetyl cysteine, grape seed extract, resveratrol, turmeric root extract, green tea leaf, and Pycnogenol (patented French Maritime Pine Bark extract, sp Pinus pinaster, Horphag Research, Geneva, Switzerland). The placebo was an identical appearing canola oil softgel. Both test formula and placebo were distributed by a masked investigator. Bottles were labelled with a unique identification number for each subject and supplied by ZeaVision, LLC, Chesterfield, Missouri, USA.

\section{METHODS}

All participants were required to read and sign informed consent, including study protocol and risks/benefits of participation, as approved by the Western Institutional Review Board (Olympia, Washington, USA). With the exception of a single, daily multivitamin and mineral (MVM) supplement (23 of 67 subjects), no subjects were currently using dietary supplements containing ingredients found in the test formula, and all agreed to refrain from using additional supplements containing any of these ingredients during the study period. Subjects also agreed to refrain from consuming xanthophyll-rich or omega-3 fatty acid-rich foodstuffs (spinach, kale, collard greens and cold water fatty fish) more than twice per week during the study period.

All subjects underwent thorough case history and comprehensive dilated eye examination prior to study enrolment, including 3 -field digital retinal photography of each eye (Kowa Optimed, Tokyo, JP). After determining study eligibility and obtaining informed consent, subjects returned within 2 weeks for the initial studyvisit consisting of the following monocular tests: contrast sensitivity was measured for spatial frequencies of 1.5 , 3, 6, 12 and 18 cycles per degree (M\&S Technologies Smart System, Skokie, Illinois, USA); macular pigment optical density (MPOD) using heterochromic flicker photometry (QuantifEye, ZeaVision, LLC, Chesterfield, Missouri, USA); Colour discrimination (L'Anthony Desaturated Color Cap test, scored via WEB-Based Scoring Software, Bela Torok, MD, PhD; accessed at http://www.torok.info/colorvision/d15.htm) and 5-2 macular threshold perimetry (Kowa Optimed, Tokyo, JP).

We assessed peripheral neuropathic symptomatology via a validated screening tool (figure 1). Within 10 days of the initial study visit, we obtained laboratory values for glycosylated haemoglobin, serum lipids (total cholesterol, low-density lipoprotein cholesterol (LDL-C), high-density lipoprotein cholesterol (HDL-C) and triglycerides), 25-OH-vitamin D3, high-sensitivity $\mathrm{C}$ reactive protein (hsCRP) for all subjects and tumour necrosis factor $\alpha(\mathrm{TNF}-\alpha)$ for subjects with retinopathy at baseline only. Foveal and mean retinal nerve fibre layer (RNFL) thickness was measured using SDOCT (Cirrus-SDOCT, Carl Zeiss, Peabody, Massachusetts, USA).

Participants were randomised to receive sequentially numbered vials of either test formula or placebo by creation of a

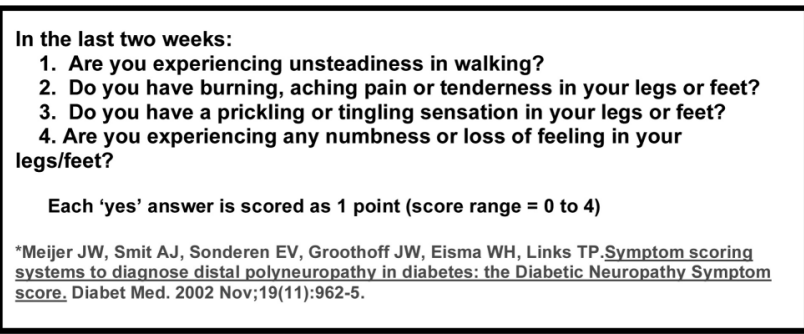

Figure 1 Diabetic Peripheral Neuropathy Symptom Score (DPNSS). 
A

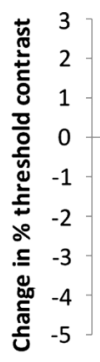

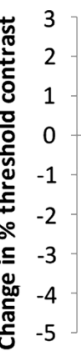

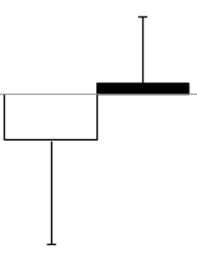

$O D$

ธSupplement - Placebo

OS

$p<0.01$

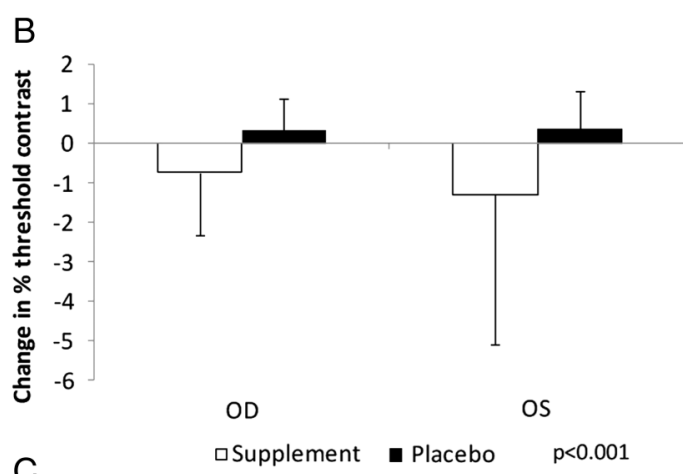

C

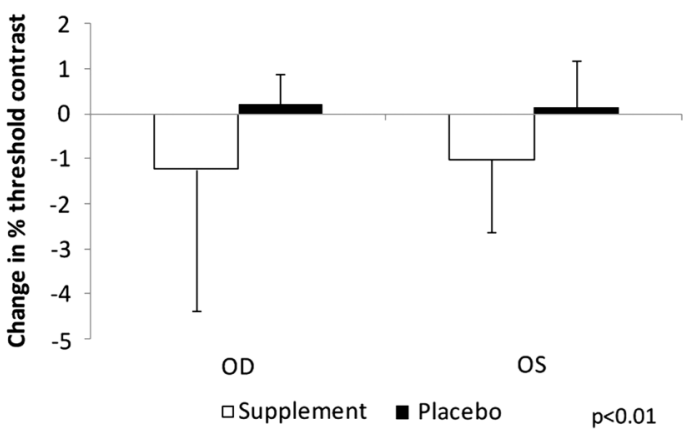

D

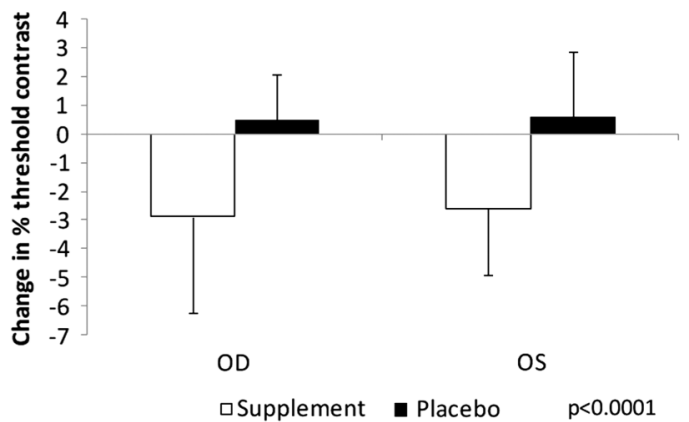

E

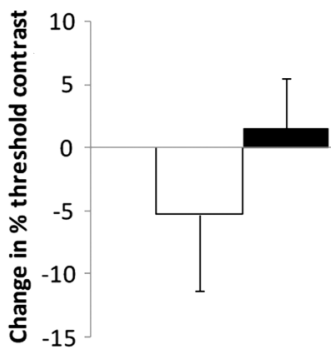

OD

口Supplement

Figure 2 Mean change over 6 months by eye for contrast sensitivity function at (A) 1.5 cycles/degree (c/d), (B) $3 \mathrm{c} / \mathrm{d}$, (C) $6 \mathrm{c} / \mathrm{d},(\mathrm{D}) 12 \mathrm{c} / \mathrm{d}$ and (E) $18 \mathrm{c} / \mathrm{d}$ for supplement and placebo groups. OD, right eye; OS, left eye.

blocked randomisation list (https://www.sealedenvelope.com/ simple-randomiser/v1/lists) according to predetermined ratios for subjects with and without DR (2:1 ratio for subjects without

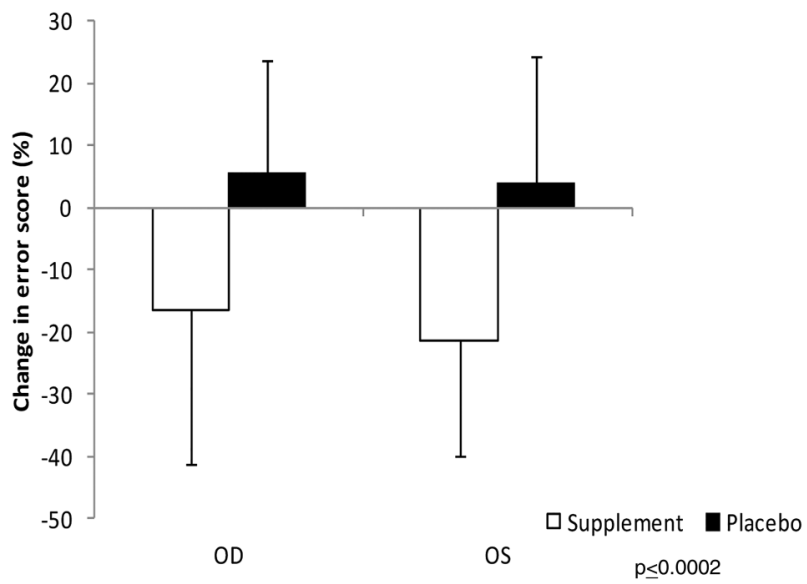

A higher color error score denotes less normal/more abnormal color discrimination

Figure 3 Mean change over 6 months for total colour error score by eye for supplement and placebo groups. A higher colour error score denotes less normal/more abnormal colour discrimination.
DR at baseline and 1:1 ratio for subjects with DR at baseline) at a dose of two capsules per day for 6 months. Investigators and subjects alike were blinded to subjects' supplement status. Subjects returned for a pill count at 10-12 weeks after initiation to help ensure adherence. After 6 months, subjects returned for

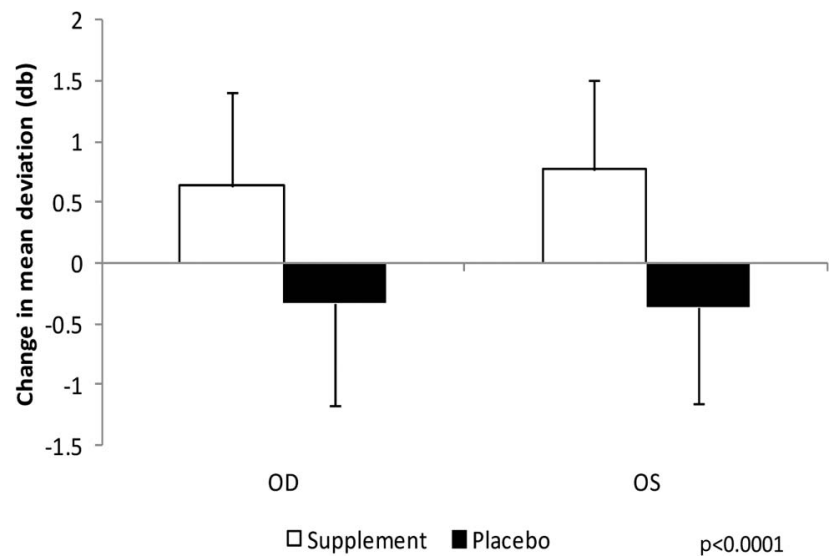

Figure 4 Mean change over 6 months in five to two threshold visual field mean deviation by eye for supplement and placebo groups. 
a final studyvisit including all tests performed at baseline and the initial study visit.

Outcomes were assessed by differences between and within groups at baseline and at study conclusion using mean+SDs and $t$ tests $(p<0.05)$ for continuous variables. Non-continuous variables (change in DR severity and Diabetic Peripheral Neuropathy Symptom Scores (DPNSSs)) were analysed for statistical significance by contingency tables using Fisher's exact test. Statistical modelling techniques were used to allow for the incorporation of data from both eyes in our analysis. This was accomplished using mixed linear models that accounted for the correlation between eyes within the model with the outcome of the model being the change from baseline. ${ }^{14}$

\section{RESULTS}

Baseline characteristics of all subjects, as well as subjects randomised to test formula and placebo are summarised in table 1 . No statistically significant differences were found at baseline between supplement and placebo groups based on age, diabetes duration, gender, diabetes subtype, DR status or use of background MVM supplements. However, a higher percentage of placebo subjects (54\%) had some degree of DR versus those receiving the DiVFuSS supplement (38\%) post randomisation (Fisher's exact test $=0.12$ ).

Baseline visual function (contrast sensitivity, colour error score and visual field mean sensitivity), MPOD, mean foveal and RNFL thickness and serum laboratory values (glycohaemoglobin (HbA1c), serum lipids, 25-OH-vitamin D3, hsCRP and TNF- $\alpha$ ) were all without significant difference. The only exception is as follows: borderline significantly better mean contrast sensitivity at three cycles per degree in the right eyes of subjects randomised to placebo $(\mathrm{p}=0.05)$.

At 6 months, visual function and MPOD significantly improved in the supplemented versus placebo group ( $p$ values ranging from 0.008 to $<0.0001)$. No significant changes in mean foveal or RNFL thickness were found between the groups. Mean changes in HDL-C, LDL-C and triglycerides showed significant improvement in the supplemented group ( $\mathrm{p}$ values were $0.0004,0.01$ and 0.01 , respectively). No statistically significant changes in mean $\mathrm{HbA1c}$, total cholesterol or TNF- $\alpha$ were found between the groups. Mean 25-OH-vitamin D3 levels were significantly higher $(\mathrm{p}=0.02)$ and hsCRP levels

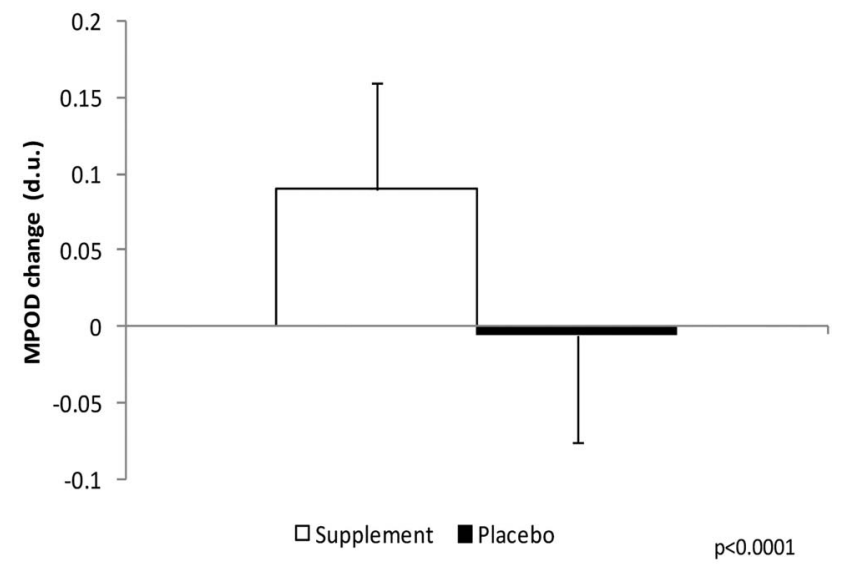

MPOD was measured in one eye at baseline and the same eye at trial completion

Figure 5 Mean change over 6 months in macular pigment optical density (MPOD) by eye for supplement and placebo groups. MPOD was measured in one eye at baseline and the same eye at trial completion.

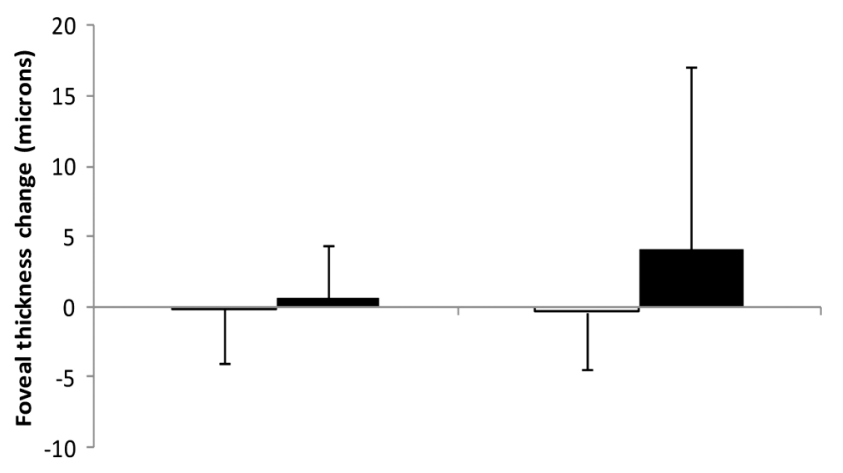

OD

OS

$\square$ Supplement $\mathbf{\square}$ Placebo $\mathrm{p}>0.05$

Figure 6 Mean change over 6 months in foveal thickness by eye for supplement and placebo groups.

were significantly lower in the supplemented group $(p=0.01)$. DPNSSs were significantly lower in the supplemented group, with one-third of supplemented subjects reporting a one unit decrease in symptoms compared with $3.6 \%$ of subjects on placebo (Fisher's exact test $\mathrm{p}=0.0024$ ).

Though not statistically significant $(\mathrm{p}=0.07)$, four subjects receiving $\mathrm{DiVFuSS}$ formula were downgraded from moderate to mild NPDR (none worsened), and one subject on placebo was upgraded to moderate NPDR (none improved). Mean changes, SDs and $\mathrm{p}$ values for assessed visual function outcomes, MPOD, OCT, HbA1c, serum lipids, 25-OH-vitamin D, TNF- $\alpha$ and hsCRP are presented in figures $2 \mathrm{~A}-\mathrm{E}$ and $3-7$ and table 2; change in DPNSS by group is presented in table 3. No adverse events were reported by any trial subject.

Using mixed linear analysis models and incorporating data from left and right eyes into a single model, where applicable, a multivariate analysis assessed by Akaike Information Criteria showed no statistically significant covariates for any outcomes of interest other than diabetes duration and contrast sensitivity at 6,12 and 18 cycles per degree $(\mathrm{p}<0.001)$.

\section{DISCUSSION}

This study demonstrates statistically significant improvement in visual function with use of a novel, multi-component nutritional supplement compared with placebo among subjects with established diabetes and/or early, NPDR. Supplemented subjects

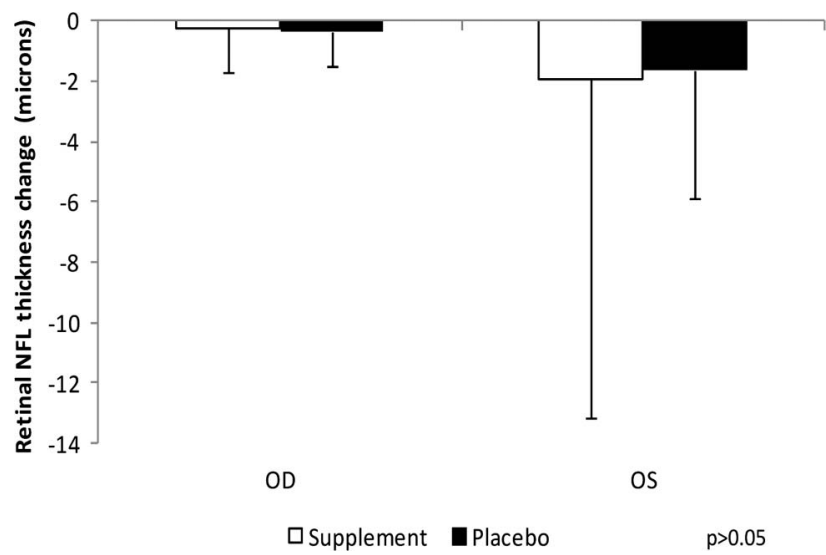

Figure 7 Mean change over 6 months in retinal nerve fibre layer by eye for supplement and placebo groups. 
Table 2 Mean change and difference in $\mathrm{HbA1C}$, total cholesterol, LDL-C, HDL-C, triglycerides, 25-OH-vitamin D, TNF- $\alpha$, hsCRP, comparing baseline to 6 months for placebo and supplement groups

\begin{tabular}{|c|c|c|c|c|c|c|c|c|}
\hline & $\mathrm{HbA} 1 \mathrm{c} \%$ & $\mathrm{TC}(\mathrm{mg} / \mathrm{dL})$ & $\begin{array}{l}\text { LDL-C } \\
(\mathrm{mg} / \mathrm{dL})\end{array}$ & $\begin{array}{l}\text { HDL-C } \\
\text { (mg/dL) }\end{array}$ & TGs (mg/dL) & $\begin{array}{l}\text { 25-OH-D } \\
\text { (ng/mL) }\end{array}$ & $\begin{array}{l}\text { TNF- } \alpha \\
(\mathrm{pg} / \mathrm{mL})\end{array}$ & $\begin{array}{l}\text { hsCRP } \\
\text { (mg/L) }\end{array}$ \\
\hline Mean change and SD for placebo group & $+0.1 \pm 0.4$ & $+0.39 \pm 13.13$ & $+0.82 \pm 10.15$ & $-1.61 \pm 5.31$ & $+2.39 \pm 11.56$ & $+0.61 \pm 7.21$ & $+0.56 \pm 2.79$ & $-0.28 \pm 1.83$ \\
\hline Mean change and SD for DiVFuSS group & $-0.1 \pm 0.4$ & $-8.33 \pm 23.40$ & $-7.61 \pm 16.08$ & $+3.82 \pm 6.24$ & $-10.46 \pm 28.48$ & $+4.54 \pm 5.93$ & $+0.78 \pm 5.04$ & $-2.14 \pm 3$ \\
\hline Mean difference between placebo and DiVFuSS groups & $0.2 \pm 0.5$ & $8.73 \pm 19.79$ & $8.44 \pm 13.93$ & $5.43 \pm 5.87$ & $12.85 \pm 23.01$ & $3.93 \pm 6.49$ & $0.22 \pm 4.08$ & $1.86 \pm 3.05$ \\
\hline $95 \% \mathrm{p}$ values & 0.06 & 0.06 & 0.01 & 0.0004 & 0.01 & 0.02 & 0.88 & 0.01 \\
\hline
\end{tabular}

25-OH-D, 25-OH-vitamin D; DiVFuSS, Diabetes Visual Function Supplement Study; HbA1c, glycohaemoglobin; HDL-C, high-density lipoprotein cholesterol; hsCRP, high-sensitivity C reactive protein; LDL-C, low-density lipoprotein cholesterol; TC, total cholesterol; TGs, triglycerides; TNF- $\alpha$, tumour necrosis factor- $\alpha$.

achieved significant increases in MPOD (27\% mean increase in DiVFuSS group vs $2 \%$ mean decrease in placebo group), as well as reductions in serum hsCRP (60\% mean decrease in DiVFuSS group vs $11 \%$ mean decrease in placebo group) and symptoms associated with diabetic peripheral neuropathy (one-third of the DiVFuSS group improved whereas only one of 28 subjects in the placebo group improved). Although serum TNF- $\alpha$ was not improved in either group, significant improvement in LDL-C (9\% mean decrease in DiVFuSS group vs $1 \%$ mean increase in placebo group), HDL-C (7\% mean increase in DiVFuSS group vs 3\% decrease in placebo group) and triglycerides $(8.6 \%$ mean decrease in DiVFuSS group vs $2 \%$ mean increase in placebo group) demonstrates positive effect on serum lipids with the test formula. The lack of effect on OCT findings is not surprising given the absence of baseline macular pathology in our study subjects combined with the relatively short duration of the trial.

The relationship between diabetic peripheral neuropathy and DR is not conclusively established, though some investigators have shown a significant, positive correlation between DR and symptomatic DPN, ${ }^{15}$ and there is overlap among the hyperglycaemia-induced molecular mediators of both disorders. ${ }^{16}$ Recent work demonstrates a direct association between increasing hsCRP and long-term risk of developing DMO in blood samples derived from type 1 diabetes subjects in the Diabetes Control and Complications Trial. This further supports the premise that the DiVFuSS nutritional intervention mitigates the pathobiology of DR. ${ }^{17}$

Age, gender, diabetes subtype, baseline retinopathy and use of a MVM during the study had no effect upon visual function enhancement observed in subjects receiving the DiVFuSS supplement versus placebo at 6 months, using multivariate analysis. The significant negative effect of diabetes duration on contrast

Table 3 Diabetic Peripheral Neuropathy Symptom Scores, change analysis comparing baseline to 6 months for placebo and supplement groups (Fisher's exact test $p=0.0024$ )

\begin{tabular}{llll}
\hline & $\begin{array}{l}\text { One-point decrease } \\
\text { (improvement) }\end{array}$ & No change & $\begin{array}{l}\text { One-point } \\
\text { increase } \\
\text { (worsening) }\end{array}$ \\
\hline $\begin{array}{l}\text { DPNSS change } \\
\text { for placebo }\end{array}$ & 1 subject (3.6\%) & $\begin{array}{l}23 \text { subjects } \\
(82.1 \%)\end{array}$ & 4 subjects (14.2\%) \\
$\begin{array}{l}\text { group } \\
\begin{array}{l}\text { DPNSS change } \\
\text { for DiVFuSS } \\
\text { group }\end{array}\end{array}$ & 13 subjects (33.3\%) & $\begin{array}{l}25 \text { subject } \\
(64.1 \%)\end{array}$ & 1 subject (2.6\%) \\
\hline $\begin{array}{l}\text { DiVFuSS, Diabetes Visual Function Supplement Study; DPNSS, Diabetic Peripheral } \\
\text { Neuropathy Symptom Score. }\end{array}$ & & \\
\hline
\end{tabular}

sensitivity between 6 and 18 cycles per degree is consistent with other studies.

Because this is a single clinical practice study, a priori sample size calculations were not performed, rather a feasible sample size was determined. The primary outcome measure was the contrast sensitivity function, determined to be statistically significant, therefore poststudy power calculations are not provided. Foveal thickness, a non-significant factor, had $80 \%$ power to detect a $6.5 \mu \mathrm{m}$ difference and $61 \%$ power to detect a $5 \mu \mathrm{m}$ difference between supplement and placebo groups in post-hoc analysis.

Diabetes causes a number of metabolic abnormalities, including hyperglycaemia, dyslipidemia, hypertension and oxidative stress. Release of inflammatory proteins, leukostasis and programmed destruction (apoptosis) of capillary endothelial cells and retinal ganglion cells may lead to breakdown of the bloodretinal barrier with vascular leakage, hypoxia and retinal neovascularisation-the hallmarks of DR (see figure 8).

Central to our understanding of diabetic microangiopathy is excess mitochondrial production of reactive oxygen species (ROS) in response to elevated intracellular glucose and nonesterified fatty acids, particularly within insulin-independent tissues. ROS inactivates the terminal enzyme, glyceraldehyde 3 phosphate dehydrogenase requisite for normal glucose metabolism, driving four distinct, upstream and injurious pathways leading to vascular compromise: protein kinase $\mathrm{C}$, hexosamine flux, advanced glycation endproduct and polyol flux. ${ }^{18}$ These pathways, in turn, augment oxidative stress, mitochondrial dysfunction and mitochondrial DNA damage in a vicious cycle that upregulates VEGF, nuclear factor kappa beta, peroxynitrite, poly-ADP ribose polymerase and a host of proinflammatory cytokines (including intercellular adhesion molecule 1 , TNF- $\alpha$, matrix metalloproteinase (MMP), interleukin-1 $\beta$, transforming growth factor- $\beta){ }^{4}$

The complex pathobiology of DR presents a number of unique challenges to investigators and clinicians alike, but yields a number of potential therapeutic targets for intervention 'downstream' from hyperglycaemia per se. In DiVFuSS, we attempted to combine micronutrients that target specific but overlapping molecular pathways implicated in the genesis of DR, based on published evidence in animal models and available human trials (see figure 9). ${ }^{4} 19$

\section{DiVFuSS formula in an animal model of DR}

Animal models have shown that single or combination antioxidant supplementation, including AREDS, can ameliorate metabolic abnormalities associated with $\mathrm{DR}$ and also prevent histopathology characteristic of DR. ${ }^{20-24}$ Of direct relevance to the present study, the multicomponent test formula used in this 


\section{PATHWAYS CONTRIBUTING TO DIABETIC RETINOPATHY}

USED WITH PERMISSION
A. PAUL CHOUS, MA, OD, FAAO

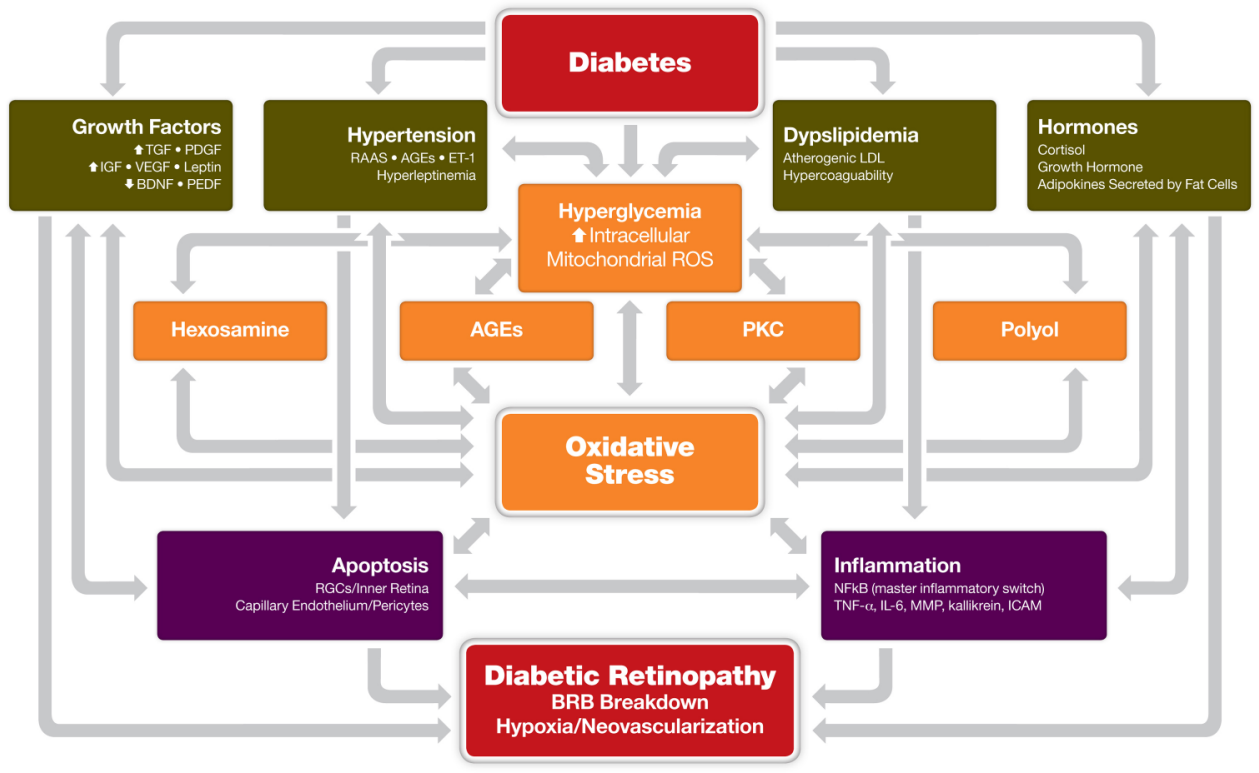

Figure 8 Biological mechanisms implicated in diabetic retinopathy. AGEs, advanced glycation endproducts; BDNF, brain-derived neurotrophic factor; BRB, blood-retinal barrier; ET-1, endothelin-1; ICAM, intercellular adhesion molecule 1; LDL, low-density lipoprotein; IGF, insulin-like growth factor; MMP, matrix metalloproteinase; NFkB, nuclear factor kappa beta; PDGF, platelet-derived growth factor; PEDF, pigment-epithelium derived factor; PKC, protein kinase C; RAAS, renin-angiotensin-aldostrerone system; RGC, retinal ganglion cell; ROS, reactive oxygen species; TGF, transforming growth factor; VEGF, vascular endothelial growth factor.

trial has been shown to attenuate diabetes-induced metabolic abnormalities in the retina, including increase in oxidative stress, mitochondrial damage and upregulation of inflammatory mediators. In addition, the test formula prevents the formation of degenerative capillaries in the retinal microvasculature, a hallmark of DR, and protects retinal function, including attenuation of decrease in the amplitudes of both $a$ - and b-waves of Electroretinogram (ERG). Thus, improvements in visual function observed in our small cohort here raises a strong possibility that this multicomponent nutritional supplement could have promising effects on the pathogenesis of retinopathy in patients with diabetes. $^{25}$

There have been few human studies stringently evaluating the effects of nutritional supplements on the development and progression of DR or its putative biomarkers. Although some studies have shown improvement in antioxidant defence mechanisms, ocular blood flow and reduction of injurious biochemical pathways, clinically meaningful endpoints such as progression to STR have been primarily assessed in observational studies rather than randomised clinical trials. Moreover, some studies have found no relationship between specific serum antioxidants (vitamins $\mathrm{C}, \mathrm{E}$ and $\beta$-carotene) and incidence/progression of DR. ${ }^{26}{ }^{27}$ This begs the question as to which specific micronutrients, or combinations thereof, are evaluated, the timing of micronutrient intervention relative to the onset of DR given the impact of metabolic memory, and the dosage or achieved concentration of any given micronutrient utilised. ${ }^{5}$

\section{CONCLUSION}

DiVFuSS demonstrates that clinically meaningful improvements in visual function can be realised with a novel, multicomponent nutritional formula in patients with both short and long duration diabetes, both type 1 and type 2 diabetes and with or without mild to moderate NPDR; couple this with significant improvements in the inflammatory serum protein, hsCRP and reduced symptoms of diabetic peripheral neuropathy-all with unperturbed glycemic control. This suggests that the DiVFuSS formula positively influences the pathogenesis of diabetes-induced retinal dysfunction with concomitant effects on visual function in a manner independent of tight or improved blood glucose control. Future studies to validate these effects both over time and in more severe DR seem warranted.

Limitations of this study include the fact that the trial was conducted at a single centre, and that serum levels of the formula's various constituents were not measured to ensure bioavailability and/or subject compliance. However, measures of pretrial and post-trial serum vitamin D status and MPOD indicate increased $25-\mathrm{OH}$ vitamin $\mathrm{D}$ and retinal uptake of the xanthophyll pigments, lutein and zeaxanthin, respectively. The short duration of the trial relative to the chronicity of diabetes, DR and their progression over time warrants longer evaluation. The inclusion of multiple components in the test formula, though theoretically targeting multiple and overlapping mechanisms implicated in DR, precludes strict analysis in the present study of synergistic or inhibitory constituent effects.

Without question, prevention of diabetes, good metabolic control combined with routine fundus examination subsequent to diagnosis and timely treatment of STR are the foundation of preventing significant vision loss associated with diabetes. Unfortunately, the prevalence of diabetes and DR continue to climb, and many patients do not achieve tight metabolic control. Although laser photocoagulation and pharmacotherapy for STR are highly effective for preventing catastrophic vision loss, these therapies are not universally effective nor without side effects; they are also expensive. DiVFuSS suggests that a novel, multicomponent formula may afford patients some 


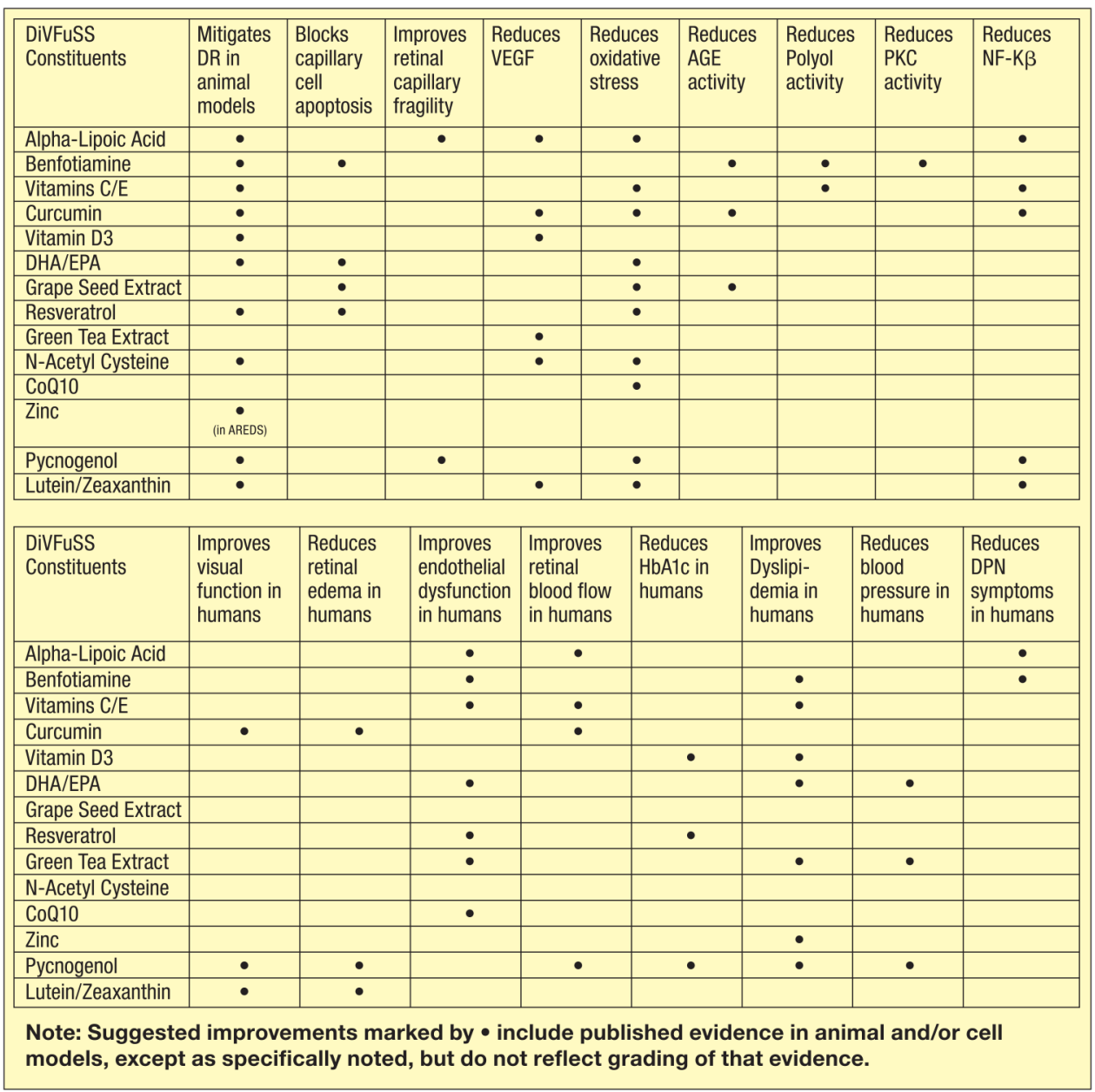

Figure 9 Rationale for inclusion of specific Diabetes Visual Function Supplement Study (DiVFuSS) micronutrients. ${ }^{4}{ }^{18}$ AGE, advanced glycation endproduct; AREDS, Age-Related Eye Disease Study; DHA/EPA, decosahexaenoic acid/eicosapentaenoic acid; DPN, Diabetic Peripheral Neuropathy; DR, diabetic retinopathy; HbA1c, glycohaemoglobin; NFkB, nuclear factor kappa beta; PKC, protein kinase C; VEGF, vascular endothelial growth factor.

protection against diminution in visual function associated with the onset and progression of DR and, potentially, the molecular and structural pathobiology of this all-too-common disorder.

Acknowledgements The authors thank Lisa Jones-Jordan and Jay M Haynie for their invaluable contributions to this study and manuscript.

Contributors APC, SPR and JDG all contributed to the design of this clinical trial. APC conducted the trial and is primarily responsible for authorship of this manuscript, with suggested clarifications and revisions made by SPR, JDG and RAK. All authors reviewed and approved the submitted manuscript, tables, figures and references.

Funding ZeaVision, LLC, Chesterfield, Missouri, USA.

Competing interests $\mathrm{APC}$, consultant to ZeaVision, LLC.

Ethics approval Western Institutional Review Board, Olympia, Washington, USA. Provenance and peer review Not commissioned; externally peer reviewed.

Open Access This is an Open Access article distributed in accordance with the Creative Commons Attribution Non Commercial (CC BY-NC 4.0) license, which permits others to distribute, remix, adapt, build upon this work non-commercially, and license their derivative works on different terms, provided the original work is properly cited and the use is non-commercial. See: http://creativecommons.org/ licenses/by-nc/4.0/

\section{REFERENCES}

1 National Eye Institute Statement. Sharp rise in diabetic eye disease makes American Diabetes Month ever more important. November 2012. http://www.nei.nih.gov/ news/statements/diabetesmonth2012.asp (accessed 8/6/13).
2 Zhang $X$, Saaddine JB, Chou CF, et al. Prevalence of diabetic retinopathy in the United States, 2005-2008. JAMA 2010;304:649-56.

3 Age-Related Eye Disease Study Research Group. A randomized, placebo-controlled, clinical trial of high-dose supplementation with vitamins $\mathrm{C}$ and $\mathrm{E}$, beta carotene, and zinc for age-related macular degeneration and vision loss: AREDS report no. 8. Arch Ophthalmol 2001;119:1417-36.

4 Kowluru RA, Zhong Q. Beyond AREDS: is there a place for antioxidant therapy in the prevention/treatment of eye disease? Invest Ophthalmol Vis Sci 2011:52:8665-71.

5 Stavrou EP, Wood JM. Letter contrast sensitivity changes in early diabetic retinopathy. Clin Exp Optom 2003:86:152-6.

6 Gualtieri M, Bandeira M, Hamer RD, et al. Contrast sensitivity mediated by inferred magno- and parvocellular pathways in type 2 diabetics with and without nonproliferative retinopathy. Invest Ophthalmol Vis Sci 2011;52:1151-5.

7 Shrestha GS, Kaiti R. Visual functions and disability in diabetic retinopathy patients. J Optom 2014;7:37-43.

8 Pinilla I, Ferreras $\mathrm{A}$, Idoipe $\mathrm{M}$, et al. Changes in frequency-doubling perimetry in patients with type I diabetes prior to retinopathy. Biomed Res Int 2013;2013:341269.

9 Giusti C. Lanthony 15-Hue Desaturated Test for screening of early color vision defects in uncomplicated juvenile diabetes. Jpn J Ophthalmol 2001;45:607-11.

10 Utku D, Atmaca LS. Farnsworth-Munsell 100-hue test for patients with diabetes mellitus. Ann Ophthalmol 1992;24:205-8.

11 Scott IU, Jackson GR, Quillen DA, et al. Effect of doxycycline vs placebo on retinal function and diabetic retinopathy progression in patients with severe nonproliferative or non-high-risk proliferative diabetic retinopathy: a randomized clinical trial. JAMA Ophthalmol 2014;132:535-43.

12 Verrotti A, Lobefalo L, Petitti MT, et al. Relationship between contrast sensitivity and metabolic control in diabetics with and without retinopathy. Ann Med 1998;30:369-74. 


\section{Clinical science}

13 Jackson GR, Scott IU, Quillen DA, et al. Inner retinal visual dysfunction is a sensitive marker of non-proliferative diabetic retinopathy. $\mathrm{Br} J$ Ophthalmol 2012;96:699-703.

14 Fan Q, Teo YY, Saw SM. Application of advanced statistics in ophthalmology. Invest Ophthalmol Vis Sci 2011;52:6059-65.

15 He BB, Wei L, Gu YJ, et al. Factors associated with diabetic retinopathy in Chinese patients with type 2 diabetes mellitus. Int J Endocrinol 2012;2012:157940.

16 Kitada M, Zhang Z, Mima A, et al. Molecular mechanisms of diabetic vascular complications. J Diabetes Investig 2010;1:77-89.

17 Muni RH, Kohly RP, Lee EQ, et al. Prospective study of inflammatory biomarkers and risk of diabetic retinopathy in the diabetes control and complications trial. JAMA Ophthalmol 2013;131:514-21.

18 Brownlee $M$. The pathobiology of diabetic complications: a unifying mechanism. Diabetes 2005;54:1615-25.

19 Chous AP. Moving the needle: can we influence the course of diabetes. Review of Optometry 2013;150:86-94.

20 Kowluru RA, Koppolu P, Chakrabarti S, et al. Diabetes-induced activation of nuclear transcriptional factor in the retina, and its inhibition by antioxidants. Free Radic Res 2003;37:1169-80.
21 Kowluru RA, Menon B, Gierhart D. Beneficial effect of zeaxanthin on retinal metabolic abnormalities in diabetic rat. Invest Ophthalmol Vis Sci 2008;49:1645-51.

22 Arnal E, Miranda M, Johnsen-Soriano S, et al. Beneficial effect of docosahexanoic acid and lutein on retinal structural, metabolic, and functional abnormalities in diabetic rats. Curr Eye Res 2009;34:928-38.

23 Hammes HP, Du X, Edelstein D, et al. Benfotiamine blocks three major pathways of hyperglycemic damage and prevents experimental diabetic retinopathy. Nat Med 2003;9:294-9.

24 Kowluru RA, Kanwar M, Chan PS, et al. Inhibition of retinopathy and retinal metabolic abnormalities in diabetic rats with AREDS-based micronutrients. Arch Ophthalmol 2008;126:1266-72.

25 Kowluru RA, Zhong Q, Santos JM, et al. Beneficial effects of the nutritional supplements on the development of diabetic retinopathy. Nutr Metab (Lond) 2014;11:8.

26 Millen $A E$, Gruber $M$, Klein $R$, et al. Relations of serum ascorbic acid and alpha-tocopherol to diabetic retinopathy in the Third National Health and Nutrition Examination Survey. Am J Epidemiol 2003;158:225-33.

27 Mayer-Davis EJ, Bell RA, Reboussin BA, et al. Antioxidant nutrient intake and diabetic retinopathy: the San Luis Valley Diabetes Study. Ophthalmology 1998; 105:2264-70. 